\title{
ÁREA SUPERFICIAL ESPECÍFICA, POROSIDADE DA FRAÇÃO ARGILA E ADSORÇÃO DE FÓSFORO EM DOIS LATOSSOLOS VERMELHOS ${ }^{(1)}$
}

\author{
Raphael Maia Aveiro Cessa ${ }^{(2)}$, Luisella Celi ${ }^{(4)}$, Antonio Carlos Tadeu \\ Vitorino $^{(3)}$, José Oscar Novelino ${ }^{(3)}$ \& Elisabetta Barberis ${ }^{(4)}$
}

\begin{abstract}
RESUMO
A porosidade da fração argila é muito importante para entendimento dos fenômenos físico-químicos que estão relacionados com a área superficial específica das partículas sólidas. Por meio de isotermas de adsorção e dessorção com $\mathrm{N}_{2}$, avaliaram-se a área superficial específica $\left(\mathrm{S}_{\mathrm{BET}}\right)$ e a porosidade das frações argila de Latossolos Vermelhos do Estado de Mato Grosso do Sul, sendo um distroférrico (LVdf) e outro distrófico (LVd), além de caulinita natural ( $\left.\mathrm{KGa}_{2}\right)$, goethita (Gt) e hematita $(\mathrm{Hm})$ sintéticas. Resultados referentes à $\mathrm{S}_{\mathrm{BET}} \mathrm{e}$ à porosidade das partículas foram relacionados à adsorção máxima de $\mathrm{P}\left(\mathrm{P}_{\text {máx }}\right)$ determinada pela isoterma de Langmuir. A classe de poros predominante nas frações argila avaliadas foi de mesoporos. As frações argila extraídas dos solos e $\mathrm{KGa}_{2}$ adsorveram quantidades semelhantes de $\mathbf{P}$ por unidade de área, mas diferentes das quantidades de $\mathbf{P}$ adsorvido por Gt e Hm. Tal diferença, possivelmente, deu-se em função da quantidade e distribuição dos grupamentos $\mathrm{OH}$ em superfície, bem como imperfeições superficiais dos materiais. Os óxidos de $\mathrm{Fe}$ e de $\mathrm{Al}$ e outros materiais amorfos das frações argila extraídas dos solos foram mais importantes que a caulinita para a adsorção de $P$. A caulinita, embora sendo o filossilicato de maior representatividade nas frações argila de LVDf e LVD, teve menor importância sobre a adsorção de $P$.
\end{abstract}

Termos de indexação: caulinita, hematita, goethita, mineralogia, argila, isoterma, fixação de fósforo.

\footnotetext{
(1) Parte da Tese de Doutorado do primeiro autor apresentada à Universidade Federal da Grande Dourados - UFGD. Recebido para publicação em julho de 2008 e aprovado em junho de 2009.

(2) Doutorando em Agronomia pela Universidade Federal da Grande Dourados - UFGD. Rodovia MS 270, km 12, Caixa Postal 533, CEP 79804-970 Dourados (MS). E-mail: raphaelcessa@hotmail.com

(3) Professor da Faculdade de Ciências Agrárias, UFGD. E-mails: vitorino@ufgd.edu.br; jnovel@ufgd.edu.br

(4) Professora da Facoltà di Agrária, Dipartamento di Valorizzazione e Protezione delle Risorse Agroforestali/Universitá di Torino. Itália. Via Leonardo da Vinci 44, Grugliasco (TO), 10095. E-mails: luisella.celi@unito.it; elisabetta.barberis@unito.it
} 


\title{
SUMMARY: SPECIFIC SURFACE ÁREA AND POROSITY OF THE CLAY FRACTION AND PHOSPHORUS ADSORPTION IN TWO RHODIC FERRALSOLS
}

\begin{abstract}
The porosity of the clay fraction is very important for the understanding of the physicochemical phenomena which are relate to the specific surface area of solid particles. The specific surface area $\left(S_{B E T}\right)$ and porosity of clay fractions of two Rhodic Ferralsols in the state of Mato Grosso do Sul, Brazil, natural kaolinite ( $\left.\mathrm{KGa}_{2}\right)$, synthetic goethite (Gt) and hematite $(\mathrm{Hm})$ were evaluated through adsorption and desorption isotherms of $N_{2}$. Results concerning $S_{B E T}$ and particle porosity were related to the maximum $P$ adsorption capacity $\left(P_{\text {max }}\right)$ estimated by the Langmuir isotherm. The predominant pore class in the clay fractions was mesopores. The clay fractions of the soils and $\mathrm{KGa}_{2}$ adsorbed similar amounts of $\mathrm{P}$ per unit area, but differed from the quantities of $P$ adsorbed by $\mathrm{Gt}$ and $\mathrm{Hm}$. This difference was possibly related to the abundance and distribution of $\mathrm{OH}$ groups on the surface as well as imperfections on the material surface. The iron and aluminum oxides and other amorphous material in the clay fractions of the soils were more important than kaolinite for Padsorption. Kaolinite, the main phyllosilicate in the clay fractions of $L V D$ f and $L V D$ soils, was less relevant for P adsorption.
\end{abstract}

Index terms: kaolinite, hemathite, goethite, mineralogy, clay, isotherm; phophorus fixation.

\section{INTRODUÇÃO}

$\mathrm{O} \mathrm{P}$ encontra-se entre os nutrientes com maior capacidade de limitar a produtividade das plantas em solos de modo geral. Sua disponibilidade nos solos continua sendo alvo de inúmeras pesquisas, envolvendo processos de adsorção e dessorção em solos tropicais intemperizados (Valladares et al., 2003; Manfredini et al., 2004), tendo em vista sua rápida passagem para formas não lábeis (Fernandes et al., 2004).

A fração argila dos Latossolos intemperizados é constituída por uma mineralogia predominantemente caulinítica, contendo hematita, goethita, e gibbsita (Melo et al., 2001; Resende et al., 2005). Segundo Brantley \& Mellott (2000), a área superficial específica de uma amostra de solo está diretamente relacionada com a mineralogia da fração argila, e ambas as propriedades em uma amostra influenciam a adsorção de P (Fontes \& Weed, 1996; Celi et al., 2003; Rolim Neto et al., 2004; Ranno et al., 2007). Com ferrihydrita pura, Weidler (1997) e Celi et al. (2003) encontraram valores elevados, 274 e $277 \mathrm{~m}^{2} \mathrm{~g}^{-1}$, respectivamente, e com hematita e goethita foram observados valores entre 30 e $46 \mathrm{~m}^{2} \mathrm{~g}^{-1}$ (Schwertmann \& Cornell, 2000; Celi et al., 2000; Prati, 2000).

A porosidade das partículas que constituem os solos influencia o tamanho da área superficial específica do solo. Microporos possuem elevada área superficial (interna) e, quando em elevada quantidade, contribuem expressivamente para aumento da área superficial específica do sólido (Greeg \& Sing, 1982). A área superficial interna é formada pelas paredes dos sulcos, poros e cavidades com profundidades maiores do que o comprimento, e com abertura para o exterior. Já a área superficial externa é constituída pela superfície dos meso e macroporos, e aumenta quando a superfície do sólido apresenta imperfeições na forma de sulcos e fissuras com dimensões maiores em comprimento do que em profundidade (Howard \& Midgley, 1981). Assim, estudos relacionados à área superficial específica de sólidos necessitam também de uma avaliação da porosidade dos sólidos, para ajudar na compreensão das possíveis variações da área superficial específica.

A contribuição dos poros para a área superficial específica pode ser avaliada por meio de isotermas de adsorção de gás (Storck et al., 1998). Com isotermas de adsorção de $\mathrm{N}_{2}$ em regiões de baixa pressão, é possível calcular a área superficial específica de um sólido, conhecendo-se o volume do gás necessário para recobrir a superfície em monocamada e a área ocupada pela molécula do gás (Greeg \& Sing, 1982).

Dessa forma, este trabalho teve por objetivo estudar a adsorção de $\mathrm{P}$ em frações argila de dois Latossolos Vermelhos do Estado de Mato Grosso do Sul, caulinita natural, goethita e hematita sintéticas, considerando a área superficial específica dos materiais como característica explicativa principal.

\section{MATERIAL E MÉTODOS}

Este estudo foi realizado no Dipartimento di Valorizzazione e Protezione delle Risorse Agroforestalle localizado na Facoltà Agrária da Università di Torino, Itália. As frações argila foram provenientes de Latossolos Vermelhos do Estado de Mato Grosso do Sul, sendo um distroférrico (LVdf) de textura muito argilosa ( $71 \%$ de argila) e outro distrófico (LVd) de textura média ( $18 \%$ de argila), localizados às margens da rodovia estadual MS-270 (Dourados - Itahum) nos $\mathrm{km} 11$ e 38, respectivamente. As amostras foram coletadas na profundidade de 20 a $40 \mathrm{~cm}$. A vegetação de cobertura do LVDf é composta predominantemente 
pela espécie Brachiaria decumbens e o LVD encontrava-se sob vegetação natural de Cerrado. Esses solos não receberam qualquer tipo de adubação há pelo menos 30 anos.

Nos quadros 1 e 2 podem ser observados os resultados das análises químicas e dos óxidos da fração argila dos solos, extraídos pelo ataque sulfúrico da terra fina seca ao ar (Embrapa, 1997).

A fração argila dos solos foi obtida por dispersão e subsequente sifonamento (Embrapa, 1997). Após secas e homogeneizadas, as frações argila foram dialisadas em membranas celulósicas com retenção máxima de 12.000 Da. Para efeito comparativo dos resultados, avaliaram-se também caulinita natural pouco cristalina $\left(\mathrm{KGA}_{2}\right)$ de Warren no Estado da Geórgia - EUA, fornecida pela Sociedade Mineral de Argila. Goethita (Gt) e hematita (Hm) foram sintetizadas segundo método de Schwertmann \& Cornell (2000).

A caracterização mineralógica qualitativa das frações argila de LVDf e LVD (Figura 1) foi obtida por meio de difratrogramas, mediante a análise em pó sem orientação dos materiais. Utilizou-se um difratômetro Philips PW1710, radiação CoKa, com condições analíticas: $1^{\circ}$ abertura divergente; $0,2^{\circ}$ abertura de entrada, intervalo de varredura $2-80^{\circ} 2 \theta$, passo de $0,02^{\circ} 2 \theta$ e passo de 1 segundo.
Os teores de $\mathrm{Fe}$ e de $\mathrm{Al}$ das formas mal cristalizadas e livres foram extraídos com oxalato ácido de amônio (Schwertmann, 1973; Jackson et al., 1986) e por ditionito-citrato-bicarbonato de sódio (DCB) (Holmigren, 1967), respectivamente, nos minerais sintéticos e nas frações argila dos Latossolos Vermelhos (Quadro 2).

A extração total dos óxidos na fração argila dos solos seguiu o método descrito em Embrapa (1997). Para $\mathrm{KGa}_{2}$, consideraram-se os valores do fornecedor,

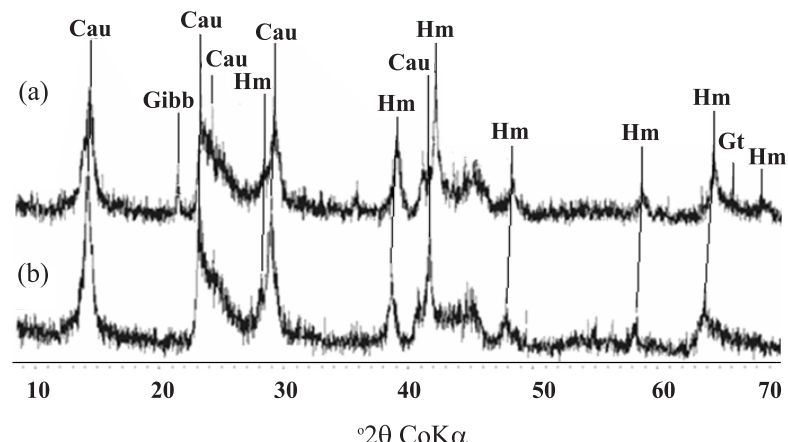

Figura 1. Difratogramas de raios X da fração argila do Latossolo Vermelho distroférrico (a) e Latossolo Vermelho distrófico (b) de Dourados, MS.

Quadro 1. Características químicas dos Latossolos Vermelhos distroférrico (LVdf) e distrófico (LVd) de Dourados (MS) coletados na profundidade de $20-40 \mathrm{~cm}$

\begin{tabular}{|c|c|c|c|c|c|c|c|c|c|c|c|c|}
\hline \multirow[b]{2}{*}{ Solo } & \multirow[b]{2}{*}{$\mathrm{CO}$} & \multirow[b]{2}{*}{$\mathbf{P}$} & \multicolumn{2}{|c|}{ pH } & \multirow[b]{2}{*}{$\mathbf{K}$} & \multirow{2}{*}{$\mathrm{Al}^{3+}$} & \multirow[b]{2}{*}{$\mathbf{C a}^{2+}$} & \multirow{2}{*}{$\mathbf{M g}^{2+}$} & \multirow{2}{*}{$\mathrm{H}+\mathrm{Al}$} & \multirow[b]{2}{*}{ SB } & \multirow[b]{2}{*}{$\mathbf{T}$} & \multirow{2}{*}{$\mathrm{V}$} \\
\hline & & & $\overline{\mathrm{H}_{2} \mathrm{O}}$ & $\mathrm{CaCl}_{2}$ & & & & & & & & \\
\hline & $\mathrm{g} \mathrm{kg}^{-1}$ & $\mathrm{mg} \mathrm{kg}^{-1}$ & & & - & - & 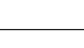 & $\mathrm{mmol}_{\mathrm{c}} \mathrm{kg}^{-1}$ & & & - & $\%$ \\
\hline LVDf & 14,3 & 1,2 & 5,2 & 4,4 & 0,4 & 15,5 & 3,6 & 1,2 & 86,4 & 5,2 & 91,6 & 5,0 \\
\hline LVD & 6,5 & 1,2 & 4,9 & 4,1 & 0,1 & 15,5 & 1,2 & 1,2 & 66,0 & 2,5 & 68,5 & 3,0 \\
\hline
\end{tabular}

CO: carbono orgânico; P: fósforo no solo extraído por Mehlich-1; $\mathrm{K}, \mathrm{Ca}, \mathrm{Mg}$ e Al: formas trocáveis; SB: soma de bases ou $\mathrm{Ca}+\mathrm{Mg}+\mathrm{K}$; T: capacidade de troca de cátions; V: saturação por bases.

Quadro 2. Teor de Fe e Al extraídos em oxalato ácido de amônio (o), ditionito-citrato-bicarbonato de sódio (d) e total em água régia (t) para a fração argila dos Latossolos Vermelhos distroférrico (LVdf) e distrófico $(\mathrm{LVd})$, caulinita natural pouco cristalina $\left(\mathrm{KGa}_{2}\right)$, goethita $(\mathrm{Gt})$ e hematita $(\mathrm{Hm})$ sintéticas

\begin{tabular}{|c|c|c|c|c|c|c|c|c|}
\hline Material & $\mathbf{F e} \mathbf{e}_{\mathrm{o}}$ & $\mathbf{F e} \mathbf{e}_{\mathrm{d}}$ & $\mathrm{Fe}_{\mathrm{t}}$ & $F e_{o} / F e_{d}$ & $\mathrm{Al}_{\mathrm{o}}$ & $\mathrm{Al}_{\mathrm{d}}$ & $\mathrm{Al}_{\mathrm{t}}$ & $\mathrm{Al}_{\mathrm{o}} / \mathrm{Al}_{\mathrm{d}}$ \\
\hline LVDf & 13,08 & 132,16 & 135,42 & 0,10 & 1,56 & 111,27 & 115,29 & 0,01 \\
\hline LVD & 14,10 & 104,35 & 106,12 & 0,14 & 4,39 & 72,63 & 102,17 & 0,06 \\
\hline $\mathrm{KGa}_{2}$ & 0,00 & 1,28 & 1,79 & 0,00 & 0,33 & 8,24 & 203,70 & 0,04 \\
\hline $\mathrm{Gt}$ & 0,55 & 590,55 & $628,59^{*}$ & 0,00 & - & - & - & - \\
\hline $\mathrm{Hm}$ & 1,08 & 634,36 & $699,44^{*}$ & 0,00 & - & - & - & - \\
\hline
\end{tabular}

*: valores calculados com base na massa molecular de Gt e Hm. 
e para Gt e Hm sintéticas foram determinados pelo cálculo das relações moleculares.

Para a construção das isotermas de adsorção de $\mathrm{P}$ foram preparadas soluções de P a 10, 100 e $1.000 \mathrm{mg} \mathrm{L}^{-1}$ em $\mathrm{KCl}$ 0,01 mol L-1 em pH 5,5. Posteriormente, em oito tubos plásticos de $10,0 \mathrm{~mL}$, foram adicionadas alíquotas das soluções de $\mathrm{P}$ em 5,0 mL de suspensões de argila, permitindo as concentrações finais de 0,0 , 2,0, 5,0, 10,0, 20,0, 30,0, 40,0, e 50,0 $\mathrm{mg} \mathrm{L}^{-1}$ de P. Em seguida, os tubos foram fechados, agitados verticalmente por $24 \mathrm{~h}$ e centrifugados a $3.000 \mathrm{rpm}$ por $15 \mathrm{~min}$. Os sobrenadantes foram passados em filtro de 0,2 $\mu \mathrm{m}$ e diluídos. As leituras para a determinação da concentração de $\mathrm{P}$ foram feitas em espectrofotômetro no comprimento de onda $600 \mathrm{~nm}$ (Braga \& Defelipo, 1974). Os teores de argila nas suspensões foram determinados de forma a permitir uma superfície específica de $\pm 10 \mathrm{~m}^{2}$ do material no tubo de $10 \mathrm{~mL}$.

O potencial zeta $(\xi)$ das frações argilosas foi determinado por velocimetria de laser Doppler $(5 \mathrm{~mW} H e-$ Ne laser 632,8 nm) acoplado a um espectroscópio de correlação de fótons, utilizando-se um espectrofotômetro Colter Delsa 440. Para isso, alíquotas das suspensões iniciais de argila eram diluídas em $\mathrm{KCl}$ $0,01 \mathrm{~mol} \mathrm{~L}^{-1}$, variando-se o valor de $\mathrm{pH}$ de 2,5 a 12,5 . Após $24 \mathrm{~h}$ de agitação, o $\xi$ foi determinado. $\mathrm{O} \mathrm{pH}$ no ponto de carga zero $\left(\mathrm{pH}_{\mathrm{PCZ}}\right)$ foi considerado o valor no qual $\xi$ foi zero.

Determinou-se a superfície específica das frações argila por meio do equipamento Sorptomatic 1900 (FISON INSTRUMENTS) capaz de fornecer os valores de adsorção e dessorção de $\mathrm{N}_{2}$ no material para construção de isotermas. Com o modelo matemático B.E.T. de Brunauer et al. (1938), foi calculada a área superficial específica dos sólidos, conhecendo-se o volume do gás necessário para recobrir em monocamada a superfície dos sólidos (Greeg \& Sing, 1982). Em geral, segundo Teixeira et al. (2001), há uma relação linear para a maioria dos sistemas adsorvente/adsorvato entre as pressões relativas $\left(p_{0-p}\right)$ 0,05 a 0,35 (região BET). Pelo método de Dubinin e Radushkevich (Greeg \& Sing, 1982), plotou-se o volume de $\mathrm{N}_{2}$ adsorvido versus a pressão relativa, obtendo-se um intercepto que serviu para estimar o volume de microporos.

Assumindo-se uma abertura de formato cilíndrica dos poros, e considerando t (espessura estatística da monocamada de $\mathrm{N}_{2}$ ) para uma pressão relativa $\left(p / p_{0}\right)$ de 0,90 , foi calculado, para faixa de mesoporos, o tamanho do poro (Sing et al., 1985). A distribuição dos tamanhos dos poros seguiu o modelo de DollimoreHeal para as classes de diâmetros: microporos $<20 \AA$, mesoporos de $20 \AA$ a $500 \AA$ e macroporos > $500 \AA$.

Com os dados das isotermas de adsorção de $\mathrm{N}_{2}$, avaliaram-se também o volume de microporos e a área superficial externa $\left(\mathrm{S}_{\mathrm{EXT}}\right)$ pelo método $\alpha$-plot (Lippens \& Boer, 1965; Sing, 1969). O valor encontrado da subtração entre superfície específica $\left(\mathrm{S}_{\mathrm{BET}}\right)$ e $\mathrm{S}_{\mathrm{EXT}}$ foi considerado a superfície interna $\left(\mathrm{S}_{\mathrm{INT}}\right)$.

\section{RESULTADOS E DISCUSSÃO}

\section{Área superficial específica e porosidade das frações argila}

$\mathrm{O}$ valor da área superficial específica $\left(\mathrm{S}_{\mathrm{BET}}\right)$ para $\mathrm{KGa}_{2}$ degasada por $5 \mathrm{~h}$ a $60{ }^{\circ} \mathrm{C}$ (Quadro 3) corresponde àquele indicado pelo fornecedor $\left(23,50 \pm 0,06 \mathrm{~m}^{2} \mathrm{~g}^{-1}\right) \mathrm{e}$ difere do encontrado por Celi et al. (2003) e Jordi et al. (2005), de $18 \mathrm{~m}^{2} \mathrm{~g}^{-1}$. Para a Hm sintética, a $\mathrm{S}_{\mathrm{BET}}$ foi de $38,36 \mathrm{~m}^{2} \mathrm{~g}^{-1}$. Schwertmann \& Cornell (2000) observaram área superficial de $30 \mathrm{~m}^{2} \mathrm{~g}^{-1}$. As $\mathrm{S}_{\mathrm{BET}}$ para Gt sintéticas nos trabalhos de Celi et al. (2000) e Prati (2000) foram, respectivamente, 46 e $44 \mathrm{~m}^{2} \mathrm{~g}^{-1}$. Neste trabalho observaram-se valores de 35,$25 ; 41,73 ; 47,38$; e $38,35 \mathrm{~m}^{2} \mathrm{~g}^{-1}$ para os respectivos degasamento: 4,16 e $18 \mathrm{~h}$ a $40{ }^{\circ} \mathrm{C} \mathrm{e} 3 \mathrm{~h}$ a $50^{\circ} \mathrm{C}$. As frações argila extraídas dos solos apresentaram os maiores valores de área superficial, e um aumento da temperatura de 40 para $60^{\circ} \mathrm{C}$ proporcionou redução da $\mathrm{S}_{\mathrm{BET}}$.

O tempo de degasamento e a temperatura são determinantes do valor de $\mathrm{S}_{\mathrm{BET}}$ da amostra e estão relacionados com a remoção de moléculas de água da superfície, permitindo que mais moléculas de $\mathrm{N}_{2}$ adsorvam-se à superfície sólida em monocamada. Weidler (1997) notou para ferrihydrita tratada com acetona, aumento da $\mathrm{S}_{\mathrm{BET}}$ com elevação da temperatura de 25 para $50{ }^{\circ} \mathrm{C}$ devido à remoção do "contaminante" sobre a superfície.

A redução da $S_{B E T}$ das frações argila do LVDf e do LVD, em função do aumento da temperatura no degasamento, pode estar relacionada aos agregados formados por óxidos. Após extração das argilas, procedeu-se à secagem em estufa a $75^{\circ} \mathrm{C}$ até formação de placas de argila, que foram trituradas em cadinho de ágata. $\mathrm{O}$ material triturado foi passado em peneira com malha de abertura de $0,123 \mathrm{~mm}$. É possível que, com o aumento da temperatura de degasamento, agregados de diâmetro $\leq 0,123 \mathrm{~mm}$, formados por associações fracas entre partículas intermediadas por moléculas de água, tenham sido alterados ou destruídos. Isso promoveria a eliminação de microporos entre partículas nos agregados que conferiam maior $\mathrm{S}_{\mathrm{BET}}$. Nota-se em tais frações, posteriormente ao degasamento, aumento de mesoporos de acordo com a distribuição do diâmetro dos poros pelo modelo Dollimore-Heal (Figuras 2 e 3).

A distribuição do diâmetro de poros é mostrada na figura 2, segundo as classes: microporos $\varnothing<20 \AA$, mesoporos $<20 \AA \varnothing<500 \AA$ e macroporos $\varnothing>500 \AA$. Para sua interpretação, considera-se que a classe predominante de poros em um sólido é aquela onde se despendeu maior quantidade de $\mathrm{N}_{2}$ para se preencher o volume de poros com determinado diâmetro. Foi observado para $\mathrm{Hm}$, Gt e $\mathrm{KGa}_{2}$ predominância de classes de poros com diâmetros próximos a $20 \AA$. Considerando-se que a distribuição do volume de poros para tais materiais (Figura 3) indicou maior mesoporosidade, dentro dessa classe, os diâmetros dos poros situam-se próximos ao limite máximo dos 


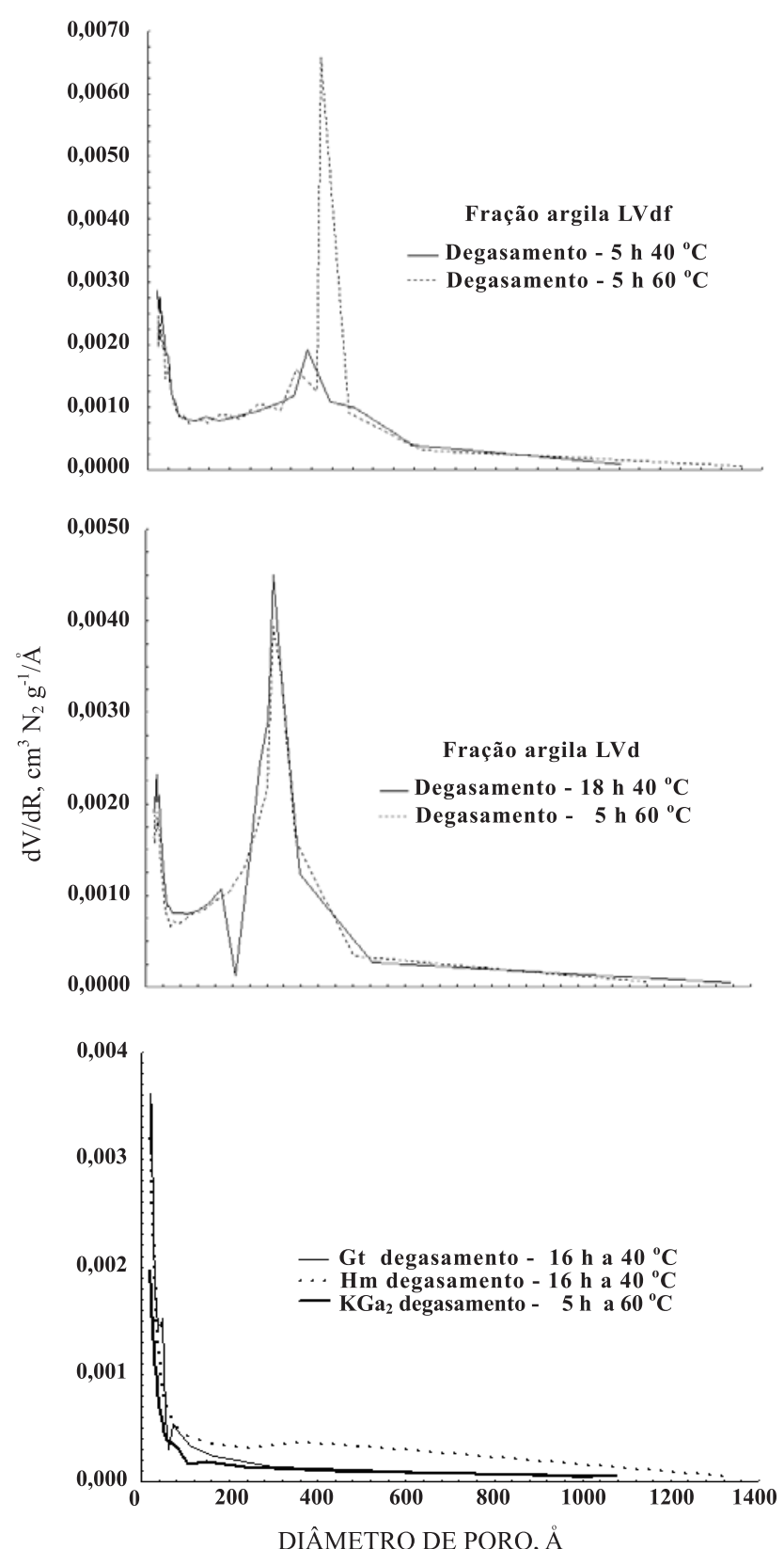

Figura 2. Distribuição do diâmetro dos poros pelo modelo Dollimore-Heal.

microporos. Para a fração argila dos solos, predominou a classe de mesoporos, porém, sobre tais frações, predominaram duas classes de mesoporos: uma próxima ao limite de microporos e outra com diâmetros entre 200 e $400 \AA$. A distribuição do diâmetro de poros da fração argila do LVDf mostrou redução da classe de macroporos com o degasamento de 40 para $60{ }^{\circ} \mathrm{C}$, aumentando dentro da subclasse de mesoporos, poros de diâmetro maior. Para a fração argila do LVD, a distribuição do tamanho de poros em função da elevação de temperatura de degasamento mostrou um comportamento semelhante, ocorrendo apenas o aparecimento de uma classe de poros com diâmetro de $200 \AA$ A para a temperatura de $60{ }^{\circ} \mathrm{C}$.

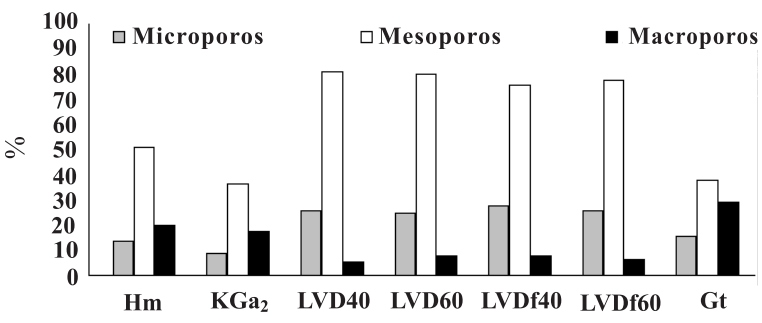

Figura 3. Volume de poros nas classes microporos: $\varnothing<20 \AA$, mesoporos: $20 \AA<\varnothing<500 \AA$, e macroporos: $500<\varnothing<1.000 \AA$, dos materiais avaliados: Hm: hematita sintética; Gt: goethita sintética; $\mathrm{KGa}_{2}$ : caulinita natural; LVDf40 LVDf60: fração argila extraída de Latossolo Vermelho distroférrico degasado a 40 e $60^{\circ} \mathrm{C}$, respectivamente; LVD40 LVD60: fração argila extraída de Latossolo Vermelho distrófico, degasado a 40 e $60^{\circ} \mathrm{C}$, respectivamente.

O formato da isoterma dá-se em função do tipo de porosidade do sólido (Brunauer, 1938). As isotermas observadas para a fração argila dos solos e $\mathrm{Hm}$ (Figura 4) assemelham-se à isoterma do tipo IV (Gregg \& Sing, 1982; Teixeira et al., 2001), característica de sólidos contendo mesoporos. A histerese natural ocorrida para essas frações é ocasionada pelo formato interno e condensamento do gás nos mesoporos, em partículas esferoidais de tamanho uniforme, compactas ou aglomeradas (Burgess et al., 1989). As isotermas para $\mathrm{KGa}_{2}$ e Gt assemelham-se à isoterma do tipo II (ausência de histerese), características de sólidos com poros razoavelmente grandes (Teixeira et al., 2001). A ausência de histerese para $\mathrm{KGa}_{2}$ e Gt possivelmente relacionou-se com a presença marcante, mas não predominante, de macroporos nesses materiais.

A presença ou ausência de histerese nos materiais pode ser explicada considerando-se o tamanho e formato dos poros, nos quais os processos de evaporação e condensação do gás são diferentes, dependendo do seu tamanho. A condensação ocorre com maior facilidade nos poros menores, onde as forças de atração são maiores devido à proximidade entre moléculas; isso pode acontecer em menores pressões (Greeg \& Sing, 1982). A evaporação, porém, é dificultada pelo formato do poro. É válido lembrar que a ausência de histerese não significa a ausência de porosidade, já que alguns formatos e tamanhos de poro podem levar a processos iguais de adsorção e dessorção.

Segundo Teixeira et al. (2001), quanto maior for o valor de $\mathrm{C}_{\mathrm{BET}}$, mais pronunciada será a inflexão na primeira região da isoterma de adsorção de $\mathrm{N}_{2}$, o que ocorrerá para sólidos microporosos. Sendo assim, maiores coeficientes de $\mathrm{C}_{\mathrm{BET}}$ (Quadro 3) e inflexões mais pronunciadas das primeiras regiões das isotermas (Figura 4) para as frações argila dos solos significam maior microporosidade nesses materiais quando comparados aos demais. $\mathrm{A} \mathrm{KGa}_{2}$ apresentou valor de $\mathrm{C}_{\mathrm{BET}}$ maior, no entanto próximo daqueles 

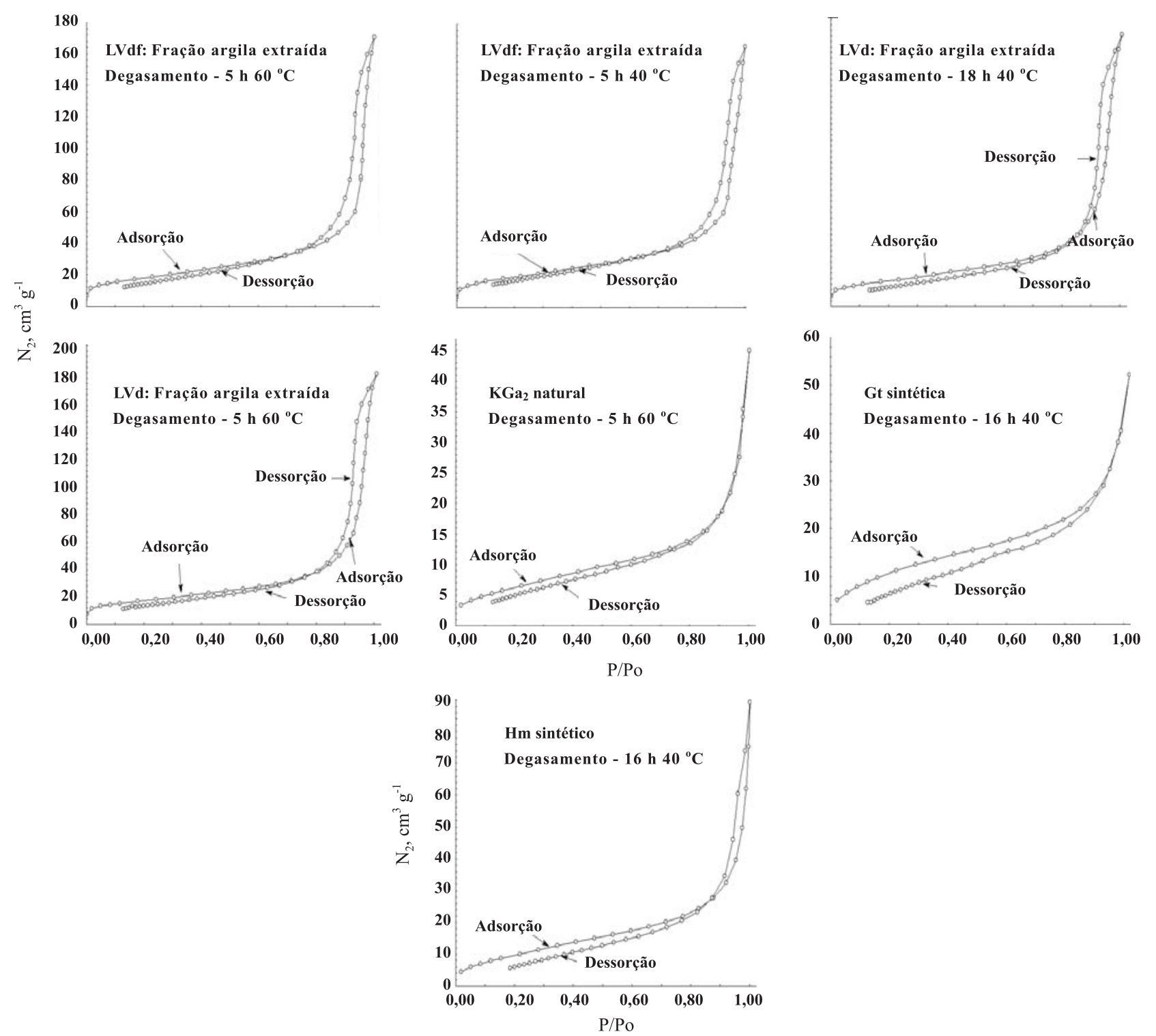

Figura 4. Isotermas de adsorção e dessorção de $\mathrm{N}_{2}$ para os materiais de argilas.

observados para Hm e Gt. Esse não foi um resultado esperado visto em menor quantidade de microporos na $\mathrm{KGa}_{2}$ em relação à Hm e Gt, porém aceitável tendo em vista a proximidade dos valores encontrados entre esses materiais nas distribuições de volume e classe de poros.

Os valores de microporosidade obtidos para os minerais por meio do modelo Dubinin \& Radushkevich (Quadro 3) foram próximos dos valores de microporosidade estimados por $\alpha$-plot, que considerou para calculo da microporosidade a região a de 1,4 a 2,4 . Nessa região, tem-se o enchimento de macroporos, porém, tratando-se de materiais com baixo volume de microporos, a extrapolação dessa região correspondeu ao volume de microporos (Echeverría et al., 1999).

Extrapolações para a região das isotermas entre o primeiro ponto e aquele ocorrido após a pressão relativa de 0,35 foram feitas para $\alpha$-plot e permitiram inferências sobre o tipo de porosidade nos matériais segundo a forma assumida para $\alpha-$ plot (Storck et al., 1998). Os $\alpha$-plot para a fração de argila do LVDf e do LVD tiveram formatos característicos de materiais mesoporosos, confirmando os resultados obtidos com o formato das isotermas de adsorção de $\mathrm{N}_{2}$ e distribuição de volume e diâmetro de poros. $\mathrm{O} \alpha$-plot para $\mathrm{KGa}_{2}$, Gt e Hm assume "levemente" um formato de materiais microporosos. Isso é possível considerando a distribuição do volume de poros para esses materiais (maior presença de microporos em relação à porosidade total), e os diâmetros de poros predominantes nesses materiais, próximos do limite dos microporos (Figuras 2 e 3 ).

\section{Adsorção de fósforo}

Com os dados de adsorção ajustados ao modelo de Langmuir, determinou-se a máxima adsorção de $\mathrm{P}$ 
Quadro 3. Área superficial específica $\left(S_{\mathrm{BET}}\right)$, externa $\left(\mathrm{S}_{\mathrm{EXT}}\right)$, interna $\left(\mathrm{S}_{\mathrm{INT}}\right)$, volume total de poros $(\mathrm{VTP})$, microporos (Mic), diâmetro de poros $(\mathrm{Dp})$ e parâmetro $\mathrm{C}$ da equação BET $\left(\mathrm{C}_{\mathrm{BET}}\right)$ das frações argila extraídas dos Latossolos Vermelhos distroférrico (LVdf) e distrófico (LVd), caulinita natural (KGa ${ }_{2}$ ), goethita (Gt) e hematita (Hm) sintéticas

\begin{tabular}{|c|c|c|c|c|c|c|c|c|c|}
\hline Argila & Tempo de degasamento & $\mathbf{S}_{\text {BET }}$ & $\mathbf{S}_{\mathrm{EXT}}$ & $\mathrm{S}_{\text {INT }}$ & VTP & $\operatorname{Mic}^{(1)}$ & $\operatorname{Mic}^{(2)}$ & Dp & $\mathbf{C}_{\text {BET }}$ \\
\hline & & 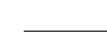 & $m^{2} g^{-1}$ & - & 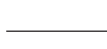 & $\mathrm{mm}^{3} \mathrm{~g}^{-1}$ & - & $\mathrm{nm}$ & \\
\hline LVDf & $5 \mathrm{~h} / 40^{\circ} \mathrm{C}$ & 67,61 & 44,62 & 22,99 & 240,12 & 27,82 & 26,80 & 24,41 & 152,61 \\
\hline LVDf & $5 \mathrm{~h} / 60^{\circ} \mathrm{C}$ & 63,55 & 35,43 & 28,12 & 247,91 & 25,69 & 27,92 & 20,88 & 145,40 \\
\hline LVD & $5 \mathrm{~h} / 40^{\circ} \mathrm{C}$ & 65,97 & 54,54 & 11,43 & 287,62 & 25,96 & 25,09 & 21,89 & 98,19 \\
\hline LVD & $5 \mathrm{~h} / 60^{\circ} \mathrm{C}$ & 60,80 & 39,46 & 21,34 & 267,16 & 24,99 & 27,04 & 20,21 & 186,86 \\
\hline Gt & $16 \mathrm{~h} / 40{ }^{\circ} \mathrm{C}$ & 41,73 & 18,92 & 22,81 & 62,50 & 15,64 & 18,11 & 21,88 & 29,95 \\
\hline $\mathrm{Hm}$ & $16 \mathrm{~h} / 40{ }^{\circ} \mathrm{C}$ & 38,36 & 24,24 & 14,12 & 116,34 & 13,73 & 14,18 & 21,63 & 28,91 \\
\hline $\mathrm{KGa}_{2}$ & $5 \mathrm{~h} / 60^{\circ} \mathrm{C}$ & 24,04 & 14,01 & 6,03 & 54,72 & 8,81 & 8,37 & 26,83 & 39,45 \\
\hline
\end{tabular}

${ }^{(1)}$ Microporosidade calculada pelo método de Dubinin \& Radushkevich (Greeg \& Sing, 1982). ${ }^{(2)}$ Microporosidade calculada por meio de $\alpha$-plot.

$\left(\mathrm{P}_{\text {máx }}, \mu \mathrm{mol} \mathrm{m} \mathrm{m}^{-2}\right)$ e o valor de $\mathrm{K}\left(\mathrm{mL} \mathrm{m}^{-2}\right)$ (constante relacionada à energia de ligação entre $\mathrm{P}$ e a superfície do mineral) sobre os materiais de argila (Quadro 4). Para goethita (Gt), a Pmáx foi próxima àquela observada por Celi et al. (2000), sendo de 2,30 $\mu \mathrm{mol} \mathrm{m} \mathrm{m}^{-2}$ de $\mathrm{P}$. Já a $\mathrm{P}_{\text {máx }}$ para hematita $(\mathrm{Hm})$ foi considerada alta e próxima àquela observada para Gt, o que poderia estar relacionado com a formação de Gt na síntese da Hm (difratrograma não apresentado). Prati (2000) observou, para Hm sintetizada pelo mesmo método, $\mathrm{P}_{\text {máx }}$ de $0,80 \mu \mathrm{mol} \mathrm{m}{ }^{-2}$ de $\mathrm{P}$. O valor $0,99 \mu \mathrm{mol} \mathrm{P} \mathrm{m}^{-2}$ de $\mathrm{P}_{\text {máx }}$ para $\mathrm{KGa} 2$ foi 2,4 vezes maior do que aquele encontrado por Celi et al. (2003). A Gt sintética foi o mineral que mais adsorveu P. Fontes \& Weed (1996) observaram que a goethita, por apresentar maior densidade de $\mathrm{OH}^{-}$de coordenação simples em uma de suas faces cristalográficas (100), teve maior afinidade de ligação com íons de fosfato (Atkinson et al., 1974; Parfitt et al., 1976), sendo isso também observado para hematita em menor intensidade. Rakovan et al. (1999) constataram, na superfície de goethita, a presença média de $75 \%$ de O-ligado ao Fe em coordenação

Quadro 4. Coeficientes das isotermas (pH 5,5) de adsorção de fósforo das frações argila dos Latossolos Vermelhos distroférrico (LVdf) e distrófico ( $\mathrm{LVd})$, caulinita natural $\left(\mathrm{KGa}_{2}\right)$, hematita (Hm) e goethita (Gt) sintéticas, ajustadas pela equação de Langmuir

\begin{tabular}{lcrr}
\hline Material & $\mathbf{X}_{\max }$ & $\mathbf{K}$ & $\mathbf{R}^{2}$ \\
\hline & $\mu \mathrm{mol} \mathrm{m}^{-2} \mathrm{de} \mathrm{P}$ & $\mathrm{mL} \mathrm{m}^{-2}$ & \\
$\mathrm{LVDf}$ & 1,03 & $2,4210^{4}$ & 0,989 \\
$\mathrm{LVD}$ & 1,10 & $4,5410^{4}$ & 0,997 \\
$\mathrm{KGa}_{2}$ & 0,99 & $3,3810^{4}$ & 0,980 \\
$\mathrm{Hm}$ & 2,35 & $4,2510^{4}$ & 0,998 \\
$\mathrm{Gt}$ & 2,42 & $10,3410^{4}$ & 0,999 \\
\hline
\end{tabular}

simples, e desta porcentagem $52 \%$ estavam ligados a $\mathrm{H}^{+}$. Isso é importante para o processo de adsorção de íons, considerando a menor quantidade de $\mathrm{O}^{-}$ligado a Fe em coordenação dupla e, ou, tripla, os quais são grupamentos não reativos (Sposito, 1984). McLaughlin et al. (1981) constataram, em sistemas mineralógicos sintéticos formados por gibbsita, goethita, hematita, caulinita e caulinita, contendo Fe precipitado em superfície, por meio de correlação positiva e significativa, que a quantidade de $\mathrm{OH}$ adsorvida por unidade de área na superfície mineral influencia o aumento da adsorção de $\mathrm{P}$.

A fração argila do LVDf e do LVD tiveram valores de $\mathrm{P}_{\text {máx }}$ próximos ao observado para KGa2, e menores em comparação aos óxidos sintéticos, fato que pode estar relacionado com a distribuição e heterogeneidade dos grupamentos protonados e deprotonados em superfície, que, por sua vez, dependem, para tal estado, do valor de pH e força iônica da solução (Dzombak \& Morel, 1990).

Segundo Rajan (1975), Bárron et al. (1988) e Celi et al. (2003), a baixa adsorção de P sobre caulinita é devida ao menor número de sítios ativos, no caso $\mathrm{OH}$. Isso fez com que a caulinita, embora tenha sido o filossilicato de maior representatividade na fração argila do LVDf e do LVD, tenha menor importância sobre o fenômeno de adsorção de $\mathrm{P}$.

Comparando-se a fração argila extraída dos solos, observou-se que a fração argila do LVD foi capaz de adsorver mais $\mathrm{P}$ por área em comparação à fração argila do LVDf. Isso deve estar relacionado com a quantidade e a forma de distribuição dos grupamentos OH na superfície da fração argila desses solos (Bárron et al., 1988; Torrent et al., 1990), bem como as imperfeições dessas superfícies. Considerando que os mesoporos contribuem significativamente para aumento da área superficial específica dos sólidos (Brantley \& Mellott, 2000), e sendo essa classe de 


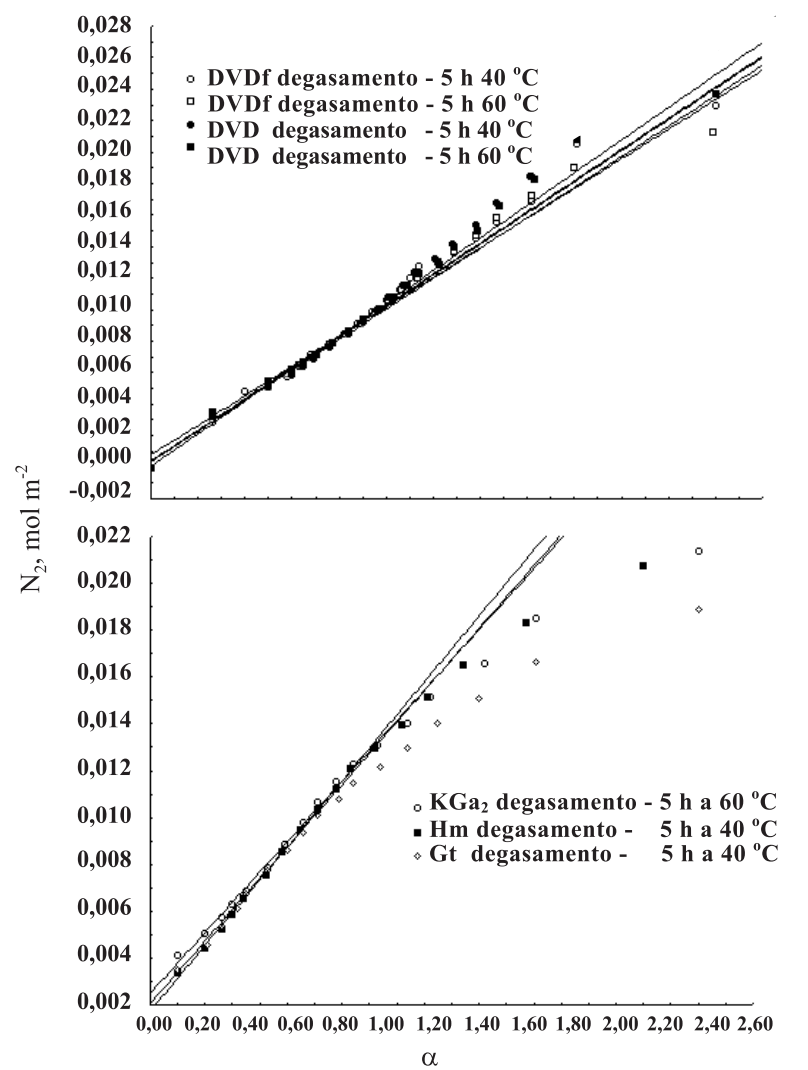

Figura 5. $\alpha$ - plot entre o primeiro ponto e aquele ocorrido após a pressão relativa de 0,35 .

poros predominante na fração argila do LVDf e do LVD, segundo Howard \& Midgley (1981), esses mesoporos seriam formados por imperfeições superficiais na forma de sulcos e fissuras com dimensões maiores em comprimento do que em profundidade.

$\mathrm{A}$ adsorção de $\mathrm{P}$ nos materiais foi também influenciada pelos valores de $\mathrm{pH}$. Para Gt e Hm, os maiores valores de $\mathrm{P}_{\text {máx }}$ observados foram atribuídos ao maior ponto de carga zero (PCZ) desses materiais (Quadro 5). Isso implica potenciais elétricos predominantemente positivos nas superfícies de Gt e $\mathrm{Hm}$, atraindo moléculas de fosfato.

Quadro 5. Valores de $\mathbf{p H}$ no ponto de carga zero $\left(\mathrm{pH}_{\mathrm{PCZ}}\right)$ da fração argila dos Latossolos Vermelhos distroférrico (LVdf) e distrófico $(\mathrm{LVd})$, caulinita natural $\left(\mathrm{KGa}_{2}\right)$, goethita $(\mathrm{Gt}) \mathrm{e}$ hematita $(\mathrm{Hm})$ sintéticas

\begin{tabular}{|c|c|c|c|}
\hline Material & $\mathbf{p H}_{\mathrm{PCZ}}$ & Minerais & $\mathbf{p H}_{\mathrm{PCZ}}$ literatura \\
\hline LVDf & 5,3 & LVDf-TFSA & $4,0^{(5)}$ \\
\hline & & & \\
\hline $\mathrm{KGa}_{2}$ & 4, & $\mathrm{Cau}$ & $4,0^{(2)} ; 4,8^{(4)} ; 5,5^{(3)}$ \\
\hline $\mathrm{Gt}$ & & & $7,0^{(2)} ;$ \\
\hline $\mathrm{Hm}$ & 6,5 & Hematita & $7,1^{(2)} ; 7,0^{(1)} ; 8,5^{(3)}$ \\
\hline \multicolumn{4}{|c|}{$\begin{array}{l}\overline{(1)} \text { Prati (2000). }{ }^{(2)} \text { Guedes et al. }(2004) .{ }^{(3)} \text { Kosmulski (2006). } \\
{ }^{(4)} \text { Kretzschmar et al. (1998). }{ }^{(5)} \text { Valor de PCZ obtido segundo } \\
\text { Camargo et al. (1986) para coleta de solo na profundidade de } \\
20-40 \mathrm{~cm} .\end{array}$} \\
\hline
\end{tabular}

\section{CONCLUSÕES}

1. A área superficial específica $\left(\mathrm{S}_{\mathrm{BET}}\right)$ das frações argila extraídas dos Latossolos Vermelhos distroférrico (LVdf) e distrófico $(\mathrm{LVd})$ foram maiores do que as $\mathrm{S}_{\mathrm{BET}}$ determinadas para caulinita natural pouco cristalina $\left(\mathrm{KGa}_{2}\right)$, goethita $(\mathrm{Gt})$ e hematita $(\mathrm{Hm})$ sintéticas. A classe de poros predominante nesses materiais estudados foi a de mesoporos.

2. As frações argila extraídas dos solos e $\mathrm{KGa}_{2}$ adsorveram quantidades semelhantes de $\mathrm{P}$ por unidade de área mas diferentes das quantidades de $\mathrm{P}$ adsorvido por Gt e Hm.

3. Os óxidos de $\mathrm{Fe}$ e de $\mathrm{Al}$, bem como outros materiais amorfos das frações argila extraídas dos solos, foram importantes para a adsorção de $\mathrm{P}$.

4. A caulinita, embora seja o filossilicato de maior representatividade nas frações argila dos solos, teve menor importância sobre a adsorção de P.

\section{LITERATURA CITADA}

ATKINSON, R.J.; PARFITT, R.L. \& SMART, R.St.C. Infrared study of phosphate adsorption on goethite. J. Chem. Soc. Faraday Trans.,70:1472-1479, 1974.

BALESDENT, J.; CHENU, C. \& BALABANE, M. Relationship of soil organic matter dynamics to physical protection and tillage. Soil Till. Res., 53:215-230, 2000.

BARRÓN, V.; HERRUZO, M. \& TORRENT, J. Phosphate adsoption by aluminous hematites of different shapes. Soil Sci. Soc. Am. J., 52:647-651, 1988.

BRAGA, J.M. \& DEFELIPO, B.V. Determinação espectrofotométrica de fósforo em extratos de solo e material vegetal. R. Ceres, 21:73-85, 1974.

BRUNAUER, S.; EMMETT, P.H. \& TELLER, E. Adsorption of gases in multimolecular layers. J. Am. Chem. Soc., 60:309319, 1938

BURGESS, C.G.V.; EVERETT, D.H. \& NUTTALL, S. Adsorption hysteresis in porous materials. Pure Appl. Chem., 61:1845-1852, 1989.

CAMARGO, O.A.; MONIZ, A.C.; JORGE, J.A. \& VALADARES, J.M.A.S. Métodos de análise química, mineralógica e física de solos do Instituto Agronômico de Campinas. Campinas, Instituto Agronômico de Campinas, 1986. 94p. (Boletim Técnico, 106)

CELI, L.; BARBERIS, E. \& MARSAN, F.A. Sorption of phosphate on goethite at high concentration. Soil Sci., 165:657-664, 2000.

CELI, L.; De LUCA, G. \& BARBERIS, E. Effects of interaction of organic and inorganic $\mathrm{P}$ with ferrihydrita and kaoliniteiron oxide systems on iron release. Soil Sci., 168:479-488, 2003. 
DZOMBAK, D.A. \& MOREL, F.M.M. Surface complexation modeling: Hydrous ferric oxide. New York, WileyInterscience, 1990. 393p.

ECHEVERRÍA, J.C.; MORERA, M.T.; MAZKIARÁN, C. \& GARRIDO, J.J. Characterization of the porous structure of soils: Adsorption of nitrogen $(77 \mathrm{~K})$ and carbon dioxide (273 K), and mercury porosimetry. Eur. J. Soil Sci., 59:497503, 1999.

EMPRESA BRASILEIRA DE PESQUISA AGROPECUÁRIA . EMBRAPA. Serviço Nacional de Levantamento e Conservação de Solos. Manual de métodos de análise de solos. 2.ed. Rio de Janeiro, 1997. 212p.

FERNANDES, R.B.A.; BARRÓN, V.; TORRENT, J. \& FONTES, M.P.F. Quantificação de óxidos de ferro de Latossolos Brasileiros por espectroscopia de refletância difusa. R. Bras. Ci. Solo, 28:245-257, 2004.

FONTES, M.P.F. \& WEED, S.B. Phosphate adsorption by clays from Brazilian Oxisols: Relationships with specifc surface area and mineralogy. Geoderma, 72:37-51, 1996.

GREGG, S.J. \& SING, K.S.W. Adsorption, surface area, and porosity. London, Academic Press, 1982. 303p.

GUEDES, C.D.; PEREIRA, J.G. \& LENA, J.C. Coagulation/ flocculation of suspensions of iron oxide particles by aluminum sulphate. Química Nova, 27:715-719, 2004.

HOLMIGREN, G.G.S. A rapid citrate dithionite extractable iron procedure. Soil Sci. Soc. Am. Proc., 31:210-211, 1967.

HOWARD, G.J. \& MIDGLEY, C.A. The formation and structure of suspension-polymerized styrene-divinylbenzene copolymers. J. Appl. Polym. Sci., 26:3845- 3870, 1981.

JACKSON, M.L.; LIM, C.H. \& ZELAZNY, L.W. Oxides, hydroxides and aluminosilicates. Section 6-3. Noncrystalline aluminosilicate and hydrous oxides by acid ammonium oxalate in the dark. In: KLUTE, A., ed. Methods of soil analysis: Physical and mineralogical methods. 2.ed. Madison, America Society of Agronomy, 1986. Part 1. p.113-118. (Agronomy, 9)

JORDI, C.; VOLKER, M. \& JIWCHAR, G. The effect of $\mathrm{pH}$ and temperature on kaolinite dissolution rate under acidi conditions. Geochim. Cosmochim. Acta, 66:3913-3926, 2005.

KOSMULSKI, M. pH - dependent surface charging and points of zero charge III. Update. J. Colloids Interface Sci., 298:730-741, 2006

KRETZSCHMAR, R.; HOLTHOFF, H. \& STICHER, H.J. Colloids Interface Sci., 202:95-103, 1998.

LIPPENS, B.C. \& De BOER, J.H. Pores systems in catalysts. V. The t-method. J. Ctalysis, 4:319-323, 1965.

MANFREDINI, D.; MELO, S.P.; TOALIARI, J.G.; FERNANDES, R.S. \& MONTEIRO, F.A. Relação silicato - fosfato em um Latossolo Vermelho-Amarelo distrófico. In: REUNIÃO BRASILEIRA DE FERTILIDADE DO SOLO E NUTRIÇÃO DE PLANTAS, 26., Lages, 2004. Anais. Lages, 2004. CD ROM

McLAUGHLIN, J.R.; RYDEN, J.C. \& SYERS, J.K. Sorption of inorganic phosphate by iron and aluminium - containing components. J. Soil Sci., 32:365-377, 1981.
MELO, V.F.; SINGH, B.; SCHAEFER, C.E.G.R.; NOVAIS, R.F. \& FONTES, M.P.F. Chemical and mineralogical properties of kaolinite - rich Brasilian soils. Soil Sci. Soc. Am. J., 65:1324-1333, 2001.

PARFITT, R.L.; FRASE, A.R. \& FARMER, V.C. Adsorption on hydrous oxides. III. Fulvic acid and humic acid on goethite, gibsite and imogolite. Eur. J. Soil Sci., 28:289-296, 1976.

PRATI, M. Adsorbimento di composti contenenti fosforo su ossidi di ferro e di titanio: Effeti sulle proprieta' di superfície. Torino, Universita Degli Studi di Torino, Università di Torino, 2000. 92p. (Tese de Mestrado)

RAJAN, S.S.S. Phosphate adsorption and the displacement of structural silicon in an allophane clay. J. Soil Sci., 26:250256,1975 .

RAKOVAN, J.; BECKER, U. \& HOCHELLA Jr., M.F. Aspects of goethite surface microtopography, structure, chemistry, and reactivity. Am. Mineral., 84:884-894, 1999.

RANNO, S. K.; SILVA, L.S.; GATIBONI, L.C. \& RHODEN, A.C. Capacidade de adsorção de fósforo em solos de várzea do Estado do Rio Grande do Sul. R. Bras. Ci. Solo, 31:21. $28,2007$.

RESENDE, M.; CURI, N.; KER, J.C. \& REZENDE, S.B. Mineralogia de solos brasileiros: Interpretação e aplicação. Lavras, Universidade Federal de Lavras, 2005. 192p.

ROLIM NETO, F.C.; SCHAEFER, C.E.G.R.; COSTA, L.M.; CORREAA, M.M.; FERNANDES FILHO, E.I.F. \& IBRAIM, M.M. Adsorção de fósforo, superfície específica e atributos mineralógicos em solos desenvolvidos de rochas vulcânicas do Alto Paranaíba (MG). R. Bras. Ci. Solo, 28:953-964, 2004

ROLIM NETO, L.M.; SEGNINI, A.; SMÕES, M.L.; MILORI, D.M.B.P.; SILVA, W.T.L. \& PRIMAVESI, O. Dinâmica e estabilidade da matéria orgânica em área com potencial para sequestro de carbono no solo. São Carlos, Embrapa Instrumentação Agropecuária, 2005. 52p. (Documentos, 17)

SCHWERTMANN, U. Use of oxalate for Fe extraction from soils. Can. J. Soil Sci., 53:244-246, 1973.

SCHWERTMANN, U. \& CORNELL, R.M. Iron oxides in the laboratory: Preparation and characterization. Second, completely revised and extendend edition. New York, Willey-VCH, 2000. 188p.

SING, K.S.W. Utilisation of adsorption data in the BET region. In: INTERNATIONAL SYMPOSIUM ON SURFACE AREA DETERMINATION, London, 1969. Proceedings. London, 1969. p.25-43

SING, K.S.W.; EVERETT, D.H.; HAUL, R.A.W.; MOSCOU, L.; PIEROTTI, R.A.; ROUQUÉROL, J. \& SIEMIENIEWSKA, T. Reporting physiosorption date for gas/solid systems with special reference to the determination of surface area and porosity. Pure Appl. Chem., 57:603-619, 1985.

SPOSITO, G. The surface chemistry of soil. New York, Oxford University Press, 1984. 234p.

STORCK, S.; BRETINGER, H. \& MAIER, W.F. Characterization of micro and mesoporous solids by physisorption methods and pore-size analysis. Appl. Catal. A: General, 174:137-146, 1998. 
TEIXEIRA, V.G.; COUTINHO, F.M.B. \& GOMES, A.S Principais métodos de caracterização da porosidade de resinas à base de divinilbenzeno. Química Nova, 24:808818, 2001.

TORRENT, J.; BÁRRON, V. \& SCHWERTMANN, U. Phosphate adsorption and desorption by goethites differing in crystal morphology. Soil Sci. Soc. Am. Proc., 36:587-593, 1990.
VALLADARES, G.S.; PEREIRA, M.G. \& ANJOS, L.H.C. Adsorção de fósforo em solos de argila de baixa atividade. Bragantia, 62:111-118, 2003.

WEIDLER, P.G. BET sample pretreatment of synthetic ferrihydrite and its influence on the determination of surface area and porosity. J. Porous Mat., 4:165$169,1997$. 